\title{
ARTICLE
}

Clinical nutrition

\section{The Refeeding Syndrome revisited: you can only diagnose what you know}

\author{
G. Janssen ${ }^{1} \cdot$ M. Pourhassan ${ }^{1} \cdot$ R. Lenzen-Großimlinghaus ${ }^{2} \cdot$ M. Jäger ${ }^{3} \cdot$ R. Schäfer ${ }^{4} \cdot$ C. Spamer SI. Cuvelier $^{6}$ • \\ D. Volkert ${ }^{7} \cdot R$. Wirth ${ }^{1} \cdot$ on behalf of the working group on nutrition and metabolism of the German \\ Geriatric Society (DGG)
}

Received: 9 April 2019 / Revised: 10 May 2019 / Accepted: 13 May 2019 / Published online: 24 May 2019

(c) The Author(s) 2019. This article is published with open access

\begin{abstract}
Background/Objectives The Refeeding Syndrome (RFS) is a serious complication in patients receiving nutrition support after a period of severe malnutrition. We frequently recognize and diagnose the RFS due to increased awareness. Thus, we observe that many physicians do not know the RFS and that it is rarely diagnosed. The aim of the study was to determine whether physicians in Germany know the RFS.

Subjects/Methods A questionnaire with a case vignette about an older person who developed the RFS after initiation of nutritional therapy was submitted to German physicians and fifth year medical students, who were participants of educational lectures.

Results Of the 281 participants who answered the respective question, 40 participants (14\%) correctly diagnosed the RFS of the case vignette and 21 participants $(8 \%)$ gave nearly correct answers. Indeed, the majority of the participants did not diagnose the RFS.

Conclusions Although the RFS may lead to fatal complications, it is unknown to the majority of the queried physicians. Therefore, there is a call to implement the RFS in respective curricula and increase systematic education on this topic.
\end{abstract}

\section{Introduction}

The Refeeding Syndrome (RFS) is a typical complication in patients receiving nutrition support after a period of severe malnutrition. The first description of this life-threatening complication was already in 1951, when Schnitker et al.

$\triangle$ R. Wirth

rainer.wirth@rub.de

1 Department for Geriatric Medicine, Marien Hospital Herne University Hospital, Ruhr-Universität Bochum, Herne, Germany

2 Ernst von Bergmann Klinikum, Potsdam, Germany

3 Hüttenhospital, Dortmund, Germany

4 GFO Kliniken Rhein-Berg, Bergisch Gladbach, Germany

5 Norden, Germany

6 Department of Geriatric Medicine, ViDia Christliche Kliniken Karlsruhe, Karlsruhe, Germany

7 Institute for Biomedicine of Aging, Friedrich-AlexanderUniversität Erlangen-Nürnberg, Nürnberg, Germany documented the course of semi-starved Japanese prisoners following liberation [1]. One fifth of these prisoners died unexpectedly despite of refeeding and vitamin supplementation. The term "refeeding syndrome" was introduced in 1981 by Weinsier and Krumdieck [2]. Up to March 2019, 1410 journal articles can be found in Medline with the search term "refeeding" and 190 journal articles are published with the search term "refeeding syndrome" in the title. However, there is no unique definition of the RFS, the prevalence of RFS is not known, and its pathophysiology is not yet completely understood. Defined by hypophosphatemia and severe symptoms occurring during the initial refeeding situation, the incidence of the RFS is suggested to be $2 \%$ among all hospital patients started on artificial nutrition [3]. Considering simply hypophosphatemia developing during the refeeding situation, the incidence of the RFS was $8 \%$ in general internal medicine [4]. The proposed mechanism of the RFS is an overwhelming transcellular shift of electrolytes during refeeding with the clinical consequences of water retention as well as a deficiency in electrolytes. Briefly, malnutrition leads to a progressive deficiency of electrolytes and micronutrients. 
Despite this intracellular deficiency, the serum level of the predominantly intracellular distributed electrolytes potassium, calcium, magnesium, and phosphate are persistently kept in balance. Therefore, a deficiency of these electrolytes cannot be diagnosed from serum levels before refeeding. Thus a sudden increase of glucose and insulin concentration after reintroduction of nutritional intake in malnourished patients causes a transcellular shift of phosphate, potassium, and magnesium from the extracellular space into the intracellular space. This transcellular shift results in a rapid decrease of serum levels with respective clinical consequences. The pathway leads to volume overload and aggravated electrolyte deficiency with a wide spectrum of conditions, including heart failure, edema, muscle spasm, rhabdomyolysis, life-threatening cardiac arrhythmia, and many other symptoms [5, 6]. Many nutrients may be involved, but low phosphate, magnesium, and potassium are suggested to be the hallmark of RFS. The syndrome typically occurs $2-5$ days after the start of refeeding and the symptoms are mostly unspecified, frequently with the clinical picture of a delirium. In general, the clinical picture may vary substantially. However, a rapid deterioration of the patient's general condition within a few days after start of the nutritional therapy is the only common characteristic. The RFS is a severe and potentially lethal complication that should be known and recognized by every physician to ensure early diagnosis and treatment and even better support preventive measures.

In 2006, the National Institute for Health and Clinical Excellence (NICE) published Guidelines about nutrition support, including criteria for the identification of patients at risk of the RFS [7]. The risk of developing RFS is suggested to be particularly high in geriatric patients. Pourhassan et al. indicated a high prevalence of risk factors of the RFS (70\%) in 342 older individuals acutely admitted in geriatric hospital units according to the NICE Guideline criteria $[8,9]$. Thus the real prevalence of the RFS is unknown [6]. Although we frequently recognize cases of RFS in our clinical routine due to increased awareness, we hypothesize that many physicians do not know the RFS, and therefore, it remains a widely unrecognized and undertreated condition.

Accordingly, the aim of this study was to determine whether physicians in Germany know the RFS and, if so, have skills in diagnosing and managing the RFS.

\section{Methods}

The study was performed as a survey. Since the term "refeeding syndrome" directly implies the basic concept of the diagnosis, we avoided to directly ask for the RFS. Alternatively, we developed a questionnaire with a typical case vignette of an older person, because the prevalence of malnutrition and of risk factors for the RFS is suggested to be particularly high in older persons. The questionnaire was submitted to German physicians and fifth year medical students, who were participants of educational lectures not dealing with nutritional medicine, in seven centers in Germany. Participation in this research was voluntary. At the beginning of each lecture, the participants were asked to directly complete the questionnaire anonymously without any aids. First, the questionnaire included questions about sex, age, medical position, years of professional experience, medical specialty, certified postgraduate education in nutritional medicine, and the federal state the participants worked in. The participants gave the answers by marking given categories with a cross. The questions about energy and fluid requirements were introduced to distract the participants from the real intention of the survey. The following case report was presented on the questionnaire and the participants had to answer a number of questions about this case:

A community dwelling, mobile, older woman had an unnoticed weight loss of $7 \mathrm{~kg}$ since her last visit to the physician's office 4 months ago. Now her body weight is $55 \mathrm{~kg}$ with a body height of $1.76 \mathrm{~m}$ (body mass index $17.8 \mathrm{~kg} / \mathrm{m}^{2}$ ). She is suffering from general weakness. Owing to arterial hypertension, she takes Ramipril and Hydrochlorothiazide in the morning. There are no other preexisting conditions. Since she is in a very good functional state, she decides to gain a differential diagnosis and a nutrition therapy.

You prescribe a $200 \mathrm{ml}$ high protein and high energy $(1.5 \mathrm{kcal} / \mathrm{ml})$ oral nutritional supplement twice a day in addition to her ordinary diet.

How do you estimate the total energy requirement of this patient? ..........kcal/day

How do you estimate the total fluid requirement of this patient? ..........ml/day

Which blood values would you check before starting the nutritional therapy?

At which time after starting the nutritional therapy would you control blood values?

Which blood values would you control?

A few days later, the general state of the patient gets worse. She develops edema, suffers from even more fatigue and weakness and seems to be mildly disoriented.

What do you suspect? Which diagnostic measures would you suggest?

Twenty-five common blood parameters, including phosphate, magnesium, and thiamine were proposed with fields to be marked by cross. In addition, the participants were able to mark the suggested time of laboratory controls as follows: during the first days, after 1 week, or 2 weeks after the start of nutrition support. For the question regarding the suspected reason for the patient's deterioration, a free text field was offered. 
The participants were asked to fill in the questionnaire immediately and supervised and without any aid. Directly afterwards, the questionnaires were collected anonymously.

The estimated energy requirements for the patient in the case report was calculated as approximately $1830 \mathrm{kcal} /$ day according to the formula of Harris-Benedict and a physical activity level of 1.6. The estimated total energy requirement was considered as correct, if it was within a range of $<15 \%$ deviation from this calculated value.

The diagnosis was considered as completely correct, if the term "refeeding syndrome" was named. It was rated as nearly correct, if there was a description of a similar state, such as electrolyte disturbance or metabolic derailment.

The selected blood values before starting the nutritional therapy were classified as completely correct if they included Sodium (Na), Potassium (K), Calcium (Ca), Magnesium $(\mathrm{Mg})$, Phosphate $(\mathrm{P})$, and Thiamine (B1). They were classified as nearly correct, if they included at least three of the six above named values, including at least phosphate. The same criteria were applied for the laboratory control after starting the nutritional therapy.

\section{Statistical analysis}

The statistical analysis was performed with the SPSS statistical software (SPSS Statistics for Windows, IBM Corp, Version 24.0, Armonk, NY, USA). Categorical variables are expressed as absolute numbers and relative frequencies (\%). Fisher's exact test was used for comparing the categorical variables with two levels, whereas categorical variables with more than two levels were compared by Chi square test. One-way analysis of variance post hoc test was performed to identify significant group differences.

\section{Results}

A total of 309 participants (59\% females) working in all 16 federal states of Germany completed the questionnaire. The majority of participants were in the age group of 21-40 years $(63 \%)$. Almost half of the population $(55 \%)$ reported a professional experience $<5$ years. Most of the responders worked in internal medicine or geriatrics $(42 \%)$. Furthermore, 12 participants (4\%) had completed a certified postgraduate course in nutritional medicine (Table 1).

Of 281 participants who answered the respective question, 40 participants (14\%) correctly diagnosed the RFS of the case vignette and 21 participants $(8 \%)$ gave nearly correct answers.

Indeed, the majority of the participants $(78 \%)$ did not diagnose the RFS or a comparable condition. As shown in Table 1, significant differences between groups could be detected. The rate of diagnosing the RFS increased with increasing age and years of medical experience. Only physicians with a professional experience $>20$ years showed a detection rate $(21 \%)$ as low as in younger physicians. Accordingly, senior physicians had a higher detection rate $(35 \%)$ than others. However, the highest rates of correct diagnosis were demonstrated by internists $(36 \%)$, geriatricians $(45 \%)$, and the participants certified in nutritional medicine $(56 \%)$.

With regard to the chosen laboratory parameter, 29 participants $(9.4 \%)$ have indicated to measure $\mathrm{Na}, \mathrm{K}, \mathrm{Ca}$, $\mathrm{Mg}, \mathrm{P}$, and Thiamine before the start of the nutritional therapy and additional 84 participants (27.4\%) recommended to measure a least phosphate.

Thirty-five participants (11.6\%) recommended to measure the above mentioned 6 parameters for control, independently of the time of control, whereas additional 52 $(17.2 \%)$ indicated to measure at least phosphate.

Sixty-two participants (20.6\%) recommended to control the blood parameter during the first days, which would have been adequate, 104 (34.6\%) after 1 week, and 135 (44.9\%) after 2 weeks.

More than half of the participants (151 out of 275; 55\%) correctly estimated the energy requirements of the patient with a significantly higher proportion in those who correctly identified the RFS (27 out of $39 ; 69 \%$ ) compared to those who did not ( 124 out of $236 ; 53 \% ; p=0.037)$. The median estimation of total fluid requirements was $1750 \mathrm{ml}$ (interquartile range: $1500-2000 \mathrm{ml}$ ) with 24 persons $(7.8 \%)$ estimating less than $1500 \mathrm{ml}$ per day.

\section{Discussion}

Our data demonstrate a huge lack of knowledge about RFS among German physicians and fifth year medical students. Only $14 \%$ diagnosed the probable RFS in the presented case vignette, with an additional $8 \%$ assuming at least a metabolic or electrolyte disturbance.

Students and young physicians were worst in recognizing the RFS. In accordance with recent studies about clinical nutrition education $[10,11]$, this is in line with the fact that there is a huge lack of clinical nutrition education in German medical schools in general and particularly regarding RFS.

The best recognition of RFS was demonstrated by participants with 10-20 years of clinical experience and by internists and geriatricians, indicating that knowledge of the RFS may predominantly be achieved by postgraduate medical education and clinical experience. Only $4 \%$ of the participants hat a certified postgraduate education in clinical nutrition, which in Germany consists of an 80-h course with 
Table 1 Characteristics of the survey participants stratified according to the recognition of refeeding syndrome (RFS)

\begin{tabular}{|c|c|c|c|c|c|}
\hline \multirow[t]{2}{*}{ Variables } & & \multicolumn{3}{|c|}{ Diagnosis of RFS } & \multirow[t]{2}{*}{$p$ Value } \\
\hline & & $n(\%)$ & $\begin{array}{l}\text { Correct and nearly correct } \\
(n=61,22 \%)\end{array}$ & $\begin{array}{l}\text { Not correct } \\
(n=220,78 \%)\end{array}$ & \\
\hline \multirow[t]{2}{*}{ Gender } & Male & $123(41.0)$ & $19(17.0)$ & $94(83.0)$ & 0.136 \\
\hline & Female & $178(59.0)$ & $40(25.0)$ & $122(75.0)$ & \\
\hline \multirow[t]{7}{*}{ Age } & $21-30$ & $130(43.0)$ & $17(15.0)$ & $97(85.0)$ & 0.011 \\
\hline & $31-40$ & $62(20.0)$ & $11(18.0)$ & $49(82.0)$ & \\
\hline & $41-50$ & $50(17.0)$ & $14(28.0)$ & $36(72.0)$ & \\
\hline & $51-60$ & $53(17.0)$ & $18(39.0)$ & $28(61.0)$ & \\
\hline & $61-70$ & $7(2.0)$ & $1(14.0)$ & $6(86.0)$ & \\
\hline & $71-80$ & $1(1.0)$ & - & - & \\
\hline & $>80$ & - & - & - & \\
\hline \multirow[t]{7}{*}{ Medical position } & Assistant physician & $91(30.0)$ & $24(28.0)$ & $62(72.0)$ & $<0.001$ \\
\hline & Senior physician & $44(15.0)$ & $14(36.0)$ & $25(64.0)$ & \\
\hline & Chief & $17(5.0)$ & $4(25.0)$ & $12(75.0)$ & \\
\hline & Private office & $25(8.0)$ & $4(16.0)$ & $21(84.0)$ & \\
\hline & Retirement & $1(1.0)$ & - & - & \\
\hline & Others & $15(5.0)$ & $8(57.0)$ & $6(43.0)$ & \\
\hline & Student & $108(36.0)$ & $4(4.0)$ & $91(96.0)$ & \\
\hline \multirow[t]{4}{*}{ Professional experience } & $<5$ & $169(55.0)$ & $21(14.0)$ & $131(86.0)$ & $<0.001$ \\
\hline & $5-10$ & $45(15.0)$ & $12(28.0)$ & $31(72.0)$ & \\
\hline & $10-20$ & $33(11.0)$ & $16(50.0)$ & $16(50.0)$ & \\
\hline & $>20$ & $58(19.0)$ & $11(21.0)$ & $41(79.0)$ & \\
\hline \multirow[t]{13}{*}{ Medical speciality } & No & $112(36.0)$ & $7(7.0)$ & $91(93.0)$ & $<0.001$ \\
\hline & General practice & $20(6.0)$ & $3(16.0)$ & $16(84.0)$ & \\
\hline & Anesthesia & $5(2.0)$ & $0(0.0)$ & $5(100.0)$ & \\
\hline & Surgery & $8(2.0)$ & $1(12.5)$ & $7(88.0)$ & \\
\hline & Geriatrics & $42(14.0)$ & $17(45.0)$ & $21(55.0)$ & \\
\hline & Gynecology & - & - & - & \\
\hline & Internal medicine & $87(28.0)$ & $31(36.0)$ & $54(64.0)$ & \\
\hline & Neurology & $11(4.0)$ & $1(14.0)$ & $6(86.0)$ & \\
\hline & Orthopedy & $15(5.0)$ & $1(7.0)$ & $13(93.0)$ & \\
\hline & Pediatric & - & - & - & \\
\hline & Psychiatry & $1(1.0)$ & $0(0.0)$ & $1(100.0)$ & \\
\hline & Urology & - & - & - & \\
\hline & Others & $5(2.0)$ & $0(0.0)$ & $5(100.0)$ & \\
\hline \multirow[t]{2}{*}{ Certified in nutritional medicine } & Yes & $12(4.0)$ & $6(56.0)$ & $5(44.0)$ & 0.014 \\
\hline & No & $285(96.0)$ & $52(20.0)$ & $210(80.0)$ & \\
\hline
\end{tabular}

The numbers of correct and not correct answers do not exactly add to the total numbers, because not all participants answered the respective question

$20 \mathrm{~h}$ of supervised clinical practice. These participants recognized the RFS significantly more frequently than others, but even here $44 \%$ did not diagnose the RFS, calling into question the respective curricula.

Although there is insufficient data about the prevalence of the RFS [12], every clinician who is aware of the RFS frequently observes it and does know about its potentially fatal consequences. In a study about the occurrence of the RFS in patients acutely admitted to an internal medicine hospital ward, $8 \%$ of the entire study population and $14 \%$ of those being considered at risk actually developed the RFS [4]. Even if only $1 \%$ of these patients would develop a severe course of the RFS $(0.08 \%)$, the prevalence would still be slightly higher than that of pulmonary embolism 
(PE) in hospital patients $(0.06 \%)$ [13], which in contrast is known by every physician. Why is the knowledge about both diseases so different? Answering this question is not a simple issue, but there are some cues to be addressed.

First, the absence of a uniform definition of the RFS may hamper systematic research and education on RFS. Second, the RFS cannot be diagnosed postmortem like PE, which may prevent physicians from correcting their underrecognition. Third, probably owing to the aforementioned issues, the RFS is completely missing in the German curricula for medical education.

Against the background that only $14-22 \%$ of physicians know the RFS, it is not justified to "throw caution to the wind" as is recommended by Matthews et al., who rarely found the RFS in the diagnoses and never found it as a primary cause of death in 260,000 hospital deaths [14]. Indeed, you can only diagnose what you know [15]. Physicians who know the RFS will prevent it or at least perform an early treatment, so that the full-blown syndrome will not develop. In addition, the development of hypophosphatemia may often not be diagnosed as RFS because no respective symptoms developed. Physicians who are not aware of the syndrome will not recognize and treat the RFS and the diagnose will never appear, neither as a simple diagnosis nor as a contributing factor to death.

Our study has some limitations. Owing to methodological reasons, the sample of participants cannot be regarded as representative for Germany. In addition, because the name Refeeding Syndrome already implies important parts of the pathophysiology, we had to enquire in an indirect way, which may have biased the answers or at least produced some inaccuracy. Nevertheless, the results give the impression that the majority of physicians do not know the RFS or are not able to apply their knowledge to a respective case vignette.

\section{Conclusion}

Although the RFS may lead to fatal complications, it is unknown to the majority of German physicians. Therefore, there is a call to implement the RFS in respective curricula and increase systematic education on this topic.

Acknowledgements We thank all members of the working group on nutrition and metabolism of the German Geriatric Society (DGG) for their valuable contribution to the study concept.

Author contributions All authors designed the study. RL-G, MJ, RS, CS, IC, and RW obtained the data. GJ, MP, and RW performed the statistical analysis. GJ, MP, DV, and RW wrote the manuscript. All authors read and approved the final version.

\section{Compliance with ethical standards}

Conflict of interest The authors declare that they have no conflict of interest.

Publisher's note: Springer Nature remains neutral with regard to jurisdictional claims in published maps and institutional affiliations.

Open Access This article is licensed under a Creative Commons Attribution 4.0 International License, which permits use, sharing, adaptation, distribution and reproduction in any medium or format, as long as you give appropriate credit to the original author(s) and the source, provide a link to the Creative Commons license, and indicate if changes were made. The images or other third party material in this article are included in the article's Creative Commons license, unless indicated otherwise in a credit line to the material. If material is not included in the article's Creative Commons license and your intended use is not permitted by statutory regulation or exceeds the permitted use, you will need to obtain permission directly from the copyright holder. To view a copy of this license, visit http://creativecommons. org/licenses/by/4.0/.

\section{References}

1. Schnitker MA, Mattman PE, Bliss TL. A clinical study of malnutrition in Japanese prisoners of war. Ann Intern Med. 1951;35:69-96.

2. Weinsier RL, Krumdieck CL. Death resulting from overzealous total parenteral nutrition: the refeeding syndrome revisited. Am J Clin Nutr. 1981;34:393-9.

3. Rio A, Whelan K, Goff L, Reidlinger DP, Smeeton N. Occurrence of refeeding syndrome in adults started on artificial nutrition support: prospective cohort study. BMJ Open. 2013;3:1.

4. Kraaijenbrink BV, Lambers WM, Mathus-Vliegen EM, Siegert $\mathrm{CE}$. Incidence of refeeding syndrome in internal medicine patients. Neth J Med. 2016;74:116-21.

5. Aubry E, Friedli N, Schuetz P, Stanga Z. Refeeding syndrome in the frail elderly population: prevention, diagnosis and management. Clin Exp Gastroenterol. 2018;11:255-64.

6. Crook MA. Refeeding syndrome: problems with definition and management. Nutrition. 2014;30:1448-55.

7. National Institute for Health and Care Excellence. Clinical guidelines. London: National Institute for Health and Care Excellence (UK); 2003. Available from: https://www.ncbi.nlm. nih.gov/books/NBK11822/.

8. Pourhassan M, Cuvelier I, Gehrke I, Marburger C, Modreker MK, Volkert D, et al. Risk factors of refeeding syndrome in malnourished older hospitalized patients. Clin Nutr. 2018;37:1354-9.

9. Pourhassan M, Cuvelier I, Gehrke I, Marburger C, Modreker MK, Volkert D, et al. Prevalence of risk factors for the refeeding syndrome in older hospitalized patients. J Nutr Health Aging. 2018;22:321-7.

10. Chung M, van Buul VJ, Wilms E, Nellessen N, Brouns FJ. Nutrition education in European medical schools: results of an international survey. Eur J Clin Nutr. 2014;68:844-6.

11. Grammatikopoulou MG, Katsouda A, Lekka K, Tsantekidis K, Bouras E, Kasapidou E, et al. Is continuing medical education sufficient? Assessing the clinical nutrition knowledge of medical doctors. Nutrition. 2019;57:69-73. 
12. Friedli N, Stanga Z, Sobotka L, Culkin A, Kondrup J, Laviano A, et al. Revisiting the refeeding syndrome: Results of a systematic review. Nutrition. 2017;35:151-60.

13. Kroger K, Kupper-Nybelen J, Moerchel C, Moysidis T, Kienitz C, Schubert I. Prevalence and economic burden of pulmonary embolism in Germany. Vasc Med. 2012;17:303-9.
14. Matthews KL, Capra SM, Palmer MA. Throw caution to the wind: is refeeding syndrome really a cause of death in acute care? Eur J Clin Nutr. 2017;72:93-8.

15. Schuetz P, Zurfluh S, Stanga Z. Mortality due to refeeding syndrome? You only find what you look for, and you only look for what you know. Eur J Clin Nutr. 2018;72:307-8. 